17.1

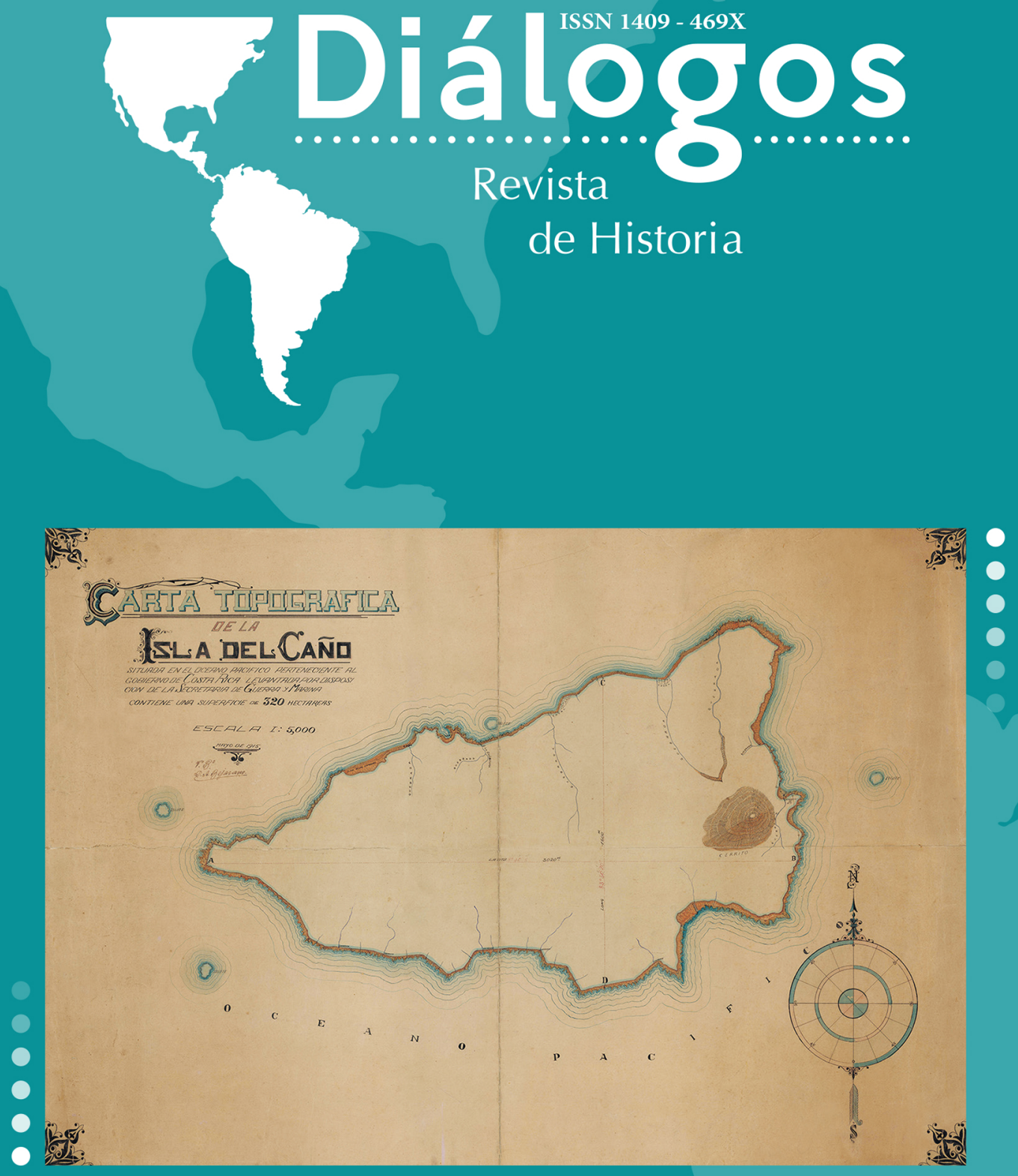

Escuela de Historia. Universidad de Costa Rica

Enero-junio 2016

url: http://revistas.ucr.ac.cr/index.php/dialogos/index 


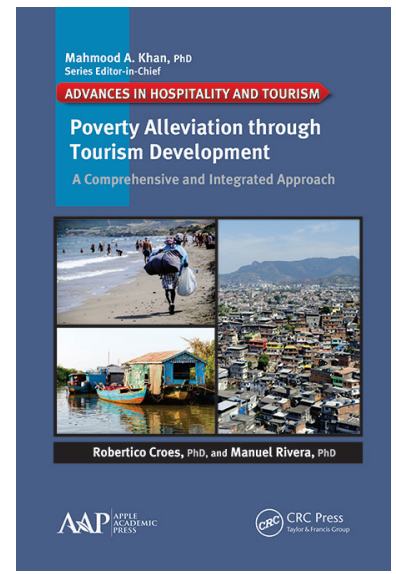

\section{Poverty Alleviation through Tourism \\ DEVELOPMENT A COMPREHENSIVE \\ AND INTEGRATED APPROACH}

Jorge Bartels Villanueva

En la gran mayoría de la literatura que aborda el estudio del turismo, los autores afirman que los efectos del turismo no contribuyen a reducir la pobreza, más bien ayuda a reforzar la desigualdad. En la propuesta de este libro, los autores discuten sobre los efectos del turismo, argumentando que este no puede automáticamente aliviar pobreza, pero su esfuerzo va dirigido a mostrar que sí se constituye en un elemento significativo para conseguir ese objetivo.

El libro "Alivio de la Pobreza a través del Desarrollo del Turismo: Un enfoque global e integrado", escrito por Robertico Croes y Manuel Rivera, ofrece un enfoque integral e integrado sobre el tema del desarrollo del turismo en los países menos desarrollados y se ofrecen evidencias empíricas de que esta actividad contribuye a la lucha contra la pobreza.

El libro es el producto de más de 8 años de investigación, realizado en países de América Latina, por medio del contacto de los investigadores con la gente pobre, los lugareños que de una forma u otra han expresado sus opiniones, sus sentimientos, su forma de ver el mundo y de cómo perciben que la actividad turística ha impacto en sus vidas. Toda esta evidencia empírica se cruza con los estudios de otros autores que también han abordado esta temática y con el material teórico expuesto en múltiples estudios sobre el turismo.

Los autores, docentes e investigadores del Rosen College of Hospitality Management, de la Universidad Central de Florida, han logrado por medio del contacto directo, a través de múltiples trabajos realizados en comunidades marginales de países pobres, en los cuales se ha pretendido desarrollar emprendimientos en la actividad turística, seguir el pulso de gente pobre, conocer sus aspiraciones, sus motivaciones y sus desencantos al vincularse con esta actividad productiva.

Fecha de recepción: 5 de noviembre de 2015 • Fecha de aceptación: 6 de noviembre de 2015

- Jorge Bartels Villanueva - Magister en Economía, Profesor e investigador, Sede del
- Pacífico de la Universidad de Costa Rica. 
El libro aborda el debate sobre el nexo turismo-crecimiento-pobreza-desigualdad, el cual se ha vuelto fuerte, amplio y polarizante a través del tiempo. Algunos autores afirman que hay una correlación positiva correlación entre crecimiento e igualdad, mientras que otros sostienen que el crecimiento económico es irrelevante para reducir la pobreza cuando los niveles de desigualdad permanecen a través del tiempo.

Mucha de la literatura sobre turismo no se ha preocupado por realizar un análisis integral de los efectos del turismo sobre los pobres y este libro es un intento en ese sentido. El tema central consiste en evaluar como el desarrollo del turismo puede impactar la pobreza, ayudando a la gente pobre a superar la línea de pobreza y a la vez cómo se puede reducir la brecha entre los ricos y los pobres de los países en menos desarrollados.

El libro consta de nueve capítulos, los que se van armando con la mira puesta en el objetivo central de la investigación, ofrecer una propuesta que vincula el desarrollo del turismo con crecimiento económico, mejores condiciones de desarrollo humano, disminución de la desigualdad y por consiguiente, reducción de la pobreza.

El primer capítulo , titulado "los rostros de los pobres", hace una revisión de la literatura que aborda el problema de la pobreza y sus dilemas, donde lo más relevante es que esa literatura muestra como la persistencia de la pobreza se asocia a tres resultados empíricos, el primero es que en los países menos desarrollados el crecimiento no es constante o persistente, lo cual hace que los ingresos no crezcan constantemente en el tiempo, segundo, los niveles de desigualdad si se mantienen a través del tiempo, constituyéndose una barrera para la eliminación de la pobreza, y tercero, en estos países la reducción de la desigualdad requiere de un compromiso social que no necesariamente existe, y que está determinado en un alto grado por la estabilidad política estabilidad y social de un país.

El capítulo 2, titulado El Paradigma de reducción de la pobreza, explora los significados y medidas de la pobreza y sus implicaciones. Esta exploración es dirigida por la pregunta: ¿cómo se pueden resolver los dilemas presentados en el capítulo anterior para la reducción de la pobreza? Para ello se hace una revisión de cuatro paradigmas utilizados en este campo, el paradigma de reducción de la pobreza vía ingresos, el enfoque de la satisfacción de las necesidades básicas, el paradigma del aumento de las capacidades de la gente pobre, y el paradigma subjetivo la pobreza. Además, en este capítulo los autores introducen su propuesta, un nuevo paradigma para reducir la pobreza, al cual han denominado: Paradigma de reducción de la pobreza (POP), por sus siglas en inglés).

El capítulo 3, donde se analiza el doble impacto potencial del desarrollo del turismo para la reducción de la pobreza. El eje central gira en torno a cómo los beneficios del turismo y el crecimiento económico favorecen a los pobres sólo cuando contribuyen a reducir la desigualdad en que estos se desenvuelven. Si el crecimiento del turismo los deja viviendo en la absoluta pobreza, cuando el crecimiento no tiene efectos sobre los pobres, se puede decir que el turismo es empobrecedor. Desde su perspectiva, el aumento de las actividades turísticas debe ir de la mano de cambio 
real de los estándares de vida. El ser pobre depende de dos factores, principalmente del nivel medio de ingresos y del grado de desigualdad en la distribución de los ingresos. Aunque la actividad turística aumente el nivel medio de los ingresos y reduzca la pobreza, cualquier aumento en desigualdad lo que hará será incrementarla.

El capítulo 4, se titula "EL poder del turismo para enfrentar la pobreza: el nexo empírico entre turismo, crecimiento, desigualdad y pobreza". Para establecer este vínculo los autores se basan en el estudios de seis casos, cinco países de América Central como son Costa Rica, El Salvador, Guatemala, Honduras, y Nicaragua. El otro caso es Ecuador ubicado en América del Sur.

Todos estos casos están relacionados con países en desarrollo, que presentan diferentes condiciones de desarrollo del turismo, y en los cuales se revela una discreta incidencia en la reducción de la pobreza. Todos estos países tienen en común que han alineado su economía con el turismo como una pieza central. Los resultados encontrados muestran que los efectos directos del turismo son evidentes, aunque estancados por la desigualdad y la debilidad de sus instituciones. También encontraron que el turismo es importante para todos los pobres de estos países, pero que los resultados varían de un país a otro. Finalmente, por medio de su análisis se llega a la conclusión de que el desarrollo del turismo constituye una estrategia efectiva para abordar los temas de la pobreza en los países en desarrollo, ya que tiene mayor impacto que otros sectores como la agricultura y la industria para el alivio de la pobreza.

El capítulo 5 se titula "Turismo e intensidad de la pobreza". El objetivo de este capítulo es analizar a profundidad los efectos del turismo sobre la gente pobre. Al evaluar la relación entre el desarrollo del turismo y la intensidad de la pobreza, es necesario examinar más de cerca los patrones del turismo y su eficacia para reducirla. El turismo puede llegar a los pobres a través de dos canales, un canal directo que ofrece ingresos a nivel individual, como los que se reciben por medio del trabajo en un hotel o un restaurante y a través de canales indirectos que son los beneficios que recibe cuando los turistas gastan en la economía y las empresas utilizan ese dinero para pagar impuestos. El gobierno recibe dinero que puede asignar programas sociales que atiendan a esa gente pobre. La principal premisa de este capítulo es que el crecimiento económico por sí solo no contribuye a erradicar la pobreza. El análisis se realiza utilizando diversos indicadores y modelos econométricos que permiten establecer las relaciones entre las variables.

"Turismo con rostro humano", es el título del capítulo 6. En este apartado se analiza la relación entre Desarrollo del turismo y Desarrollo humano, basándose en el enfoque propuesto por Armita Sen. Los autores amplían la base de información propuesta en el índice de desarrollo propuesto por Sen (índice S) e incluyen el Índice desarrollo humano (IDH) que utiliza las Naciones Unidas. En los capítulos anteriores, los autores ya habían revelado que el índice de Sen aportaba información sobre la incidencia de la pobreza, sobre cuán pobres son los pobres, y como se distribuyen los ingresos entre los pobres. Los resultados muestran la incidencia, profundidad y distribución de ingresos que se produce cuando los pobres logran aumentar 
sus ingresos. El Índice S proporciona valiosa información con respecto al papel de Turismo en cuanto beneficia a los pobres y les ayuda a elevar su nivel de ingresos, lo cual se ilustra utilizando un caso acontecido en Ecuador. Finalmente realiza una comparación de los efectos del turismo sobre el desarrollo humano en regiones de Costa Rica y Nicaragua.

El capítulo 7 se titula "El Turismo y los satisfechos pero infelices pobres". Con base en los resultados de los capítulos anteriores, los autores logran constatar que si bien La distribución de beneficios derivados de Turismo puede proporcionar recursos y ventajas a los pobres, el contar con mayores ingresos no necesariamente será utilizado para apoyar su propio crecimiento y desarrollo, es decir que el contar con más dinero no necesariamente va a hacer a la población pobre más feliz y más satisfecha con su vida. Condiciones objetivas impulsadas por el turismo, como las fuentes de trabajo no se han traducido en oportunidades o condiciones para sacar a los pobres de la pobreza. La zozobra y la incertidumbre en la cual viven los pobres se constituyen en una limitación para el mejor aprovechamiento de esas oportunidades. En este capítulo resulta muy interesante escuchar las opiniones de gente pobre, sobre cómo perciben su calidad de vida, lo que más les importa, cómo perciben el desarrollo del turismo en sus comunidades y cómo estas percepciones definen la forma como incide el turismo sobre su propio bienestar.

"Mirando a través del cristal: Desarrollo del turismo y la paradoja de la pobreza", es el título del capítulo 8. En este capítulo se parte de los hallazgos de la investigación donde se pudo constatar que aunque el turismo puede aumentar el ingresos de los pobres, esto no se traduce en una salida a la pobreza, esto porque el pobre ha experimentado como parte de los impactos de Turismo, el desplazamiento, inseguridad del trabajo, un flujo ingresos impredecible, como aspectos que limitan el poder del Turismo para luchar contra la pobreza. En este capítulo el objetivo consiste en buscar la explicación de esta yuxtapuesta realidad de desarrollo del turismo, donde se tiene pobres satisfechos pero que no tienen una vida feliz. En la investigación se logra determinar que los pobres manifiestan sentirse satisfechos con su vida, pero a la vez no se sienten felices y esto en parte se explica porque esa satisfacción es un concepto de construcción de largo plazo, mientras que la felicidad se basa en emociones y las emociones son fugaces. Cuando los pobres asocian su vida con el desarrollo del turismo, perciben el turismo como positivo y el canal a través del cual se da esta percepción positiva está articulado por las oportunidades. Pero curiosamente, cuando se considera la felicidad, esas mismas oportunidades generan una percepción negativa. El desarrollo del turismo, canalizado a través de oportunidades, parece no contribuir a superar las luchas diarias de los pobres.

El capítulo 9 y final, se titula: "Una idea loca, lo suficiente como para lograr un cambio: el Enfoque Tirón de la Demanda (DPA)". Es el resultado de todo el proceso investigativo donde los autores realizan una propuesta que sirva para dar una salida al problema de la pobreza, por medio del desarrollo del turismo, que logre un solución más consistente y de largo plazo, que permita no solo el crecimiento 
económico, el desarrollo humano, mejorar la calidad de vida y que los pobres logren empoderarse, buscar sus propias soluciones y comprender que existen oportunidades que deben ser aprovechadas para lograr superar su condición de pobreza.

Para los autores, la relación entre desarrollo del turismo y la reducción de la pobreza es, por lo tanto, un tema social complejo, contingente en un número de factores tales como motivación personal, aspiraciones, oportunidades, fortaleza institucional, el nivel de desigualdad, la posibilidad de creación de riqueza, y una clara intención de ayudar a los pobres.

Los principales predicamentos de los programas dirigidos a la reducción de la pobreza están relacionados con la presunción de que los gobiernos y las organizaciones internacionales saben lo que es mejor para los pobres. En su propuesta los autores señalan que, las libres relaciones del mercado, sin interrupción de un tercero, sea un partido de gobierno o la burocracia estatal, que se encarguen de insertar distorsiones, corrupción o desigualdad, serán las que permitan que el dinero procedente del turismo fluya a través de estas economías y pueda ser distribuido según las fuerzas del mercado. La invitación a conocer la propuesta queda planteada.

El desarrollo del turismo puede reducir pobreza. Esta reducción de en la pobreza ocurre de dos formas sobre las economías. La principal forma es por medio de los efectos directos, como es la creación de empleos y de pequeños o medianos emprendimientos que vinculan capital y trabajo. La otra forma que actúa de manera indirecta sobre la pobreza, está dada por la intervención del gobierno, que regula la actividad y realiza la gestión macroeconómica, que propicia las condiciones (inflación, empleo, acceso al crédito, etc.), y las transferencias a los pobres, por medio de su política social.

Este libro sugiere que turismo ha tenido efectos directos e indirectos como elemento para aliviar la pobreza a través de económico crecimiento. Curiosamente, los efectos directos de turismo son más fuertes que los efectos indirectos y una explicación se origina en las condiciones que deterioran los efectos indirectos producto del crecimiento económico, tales como la desigualdad y debilidad de las instituciones de los países menos desarrollados.

Con respecto a las fuentes utilizadas en el libro, destaca su variedad, cantidad y calidad. Se analizan los planteamientos teóricos sobre la pobreza, investigaciones empíricas realizadas por diversos autores en muchos países del mundo, las diferentes propuestas que se han realizado para la medición de la pobreza, tanto con planteamientos objetivos, como incorporando elementos subjetivos en la explicación de este fenómeno social, lo cual se complementa con la información que se obtuvo de diversas revistas que abordan la temática de la pobreza, todo lo cual representa una abundante bibliografía.

Otro elemento interesante de este libro lo constituye la forma en que los autores realizan el proceso investigativo, recurriendo a la colaboración de diversas personas e instituciones ubicadas en los propios países objeto de investigación, lo cual sin duda fue clave para la exitosa culminación de un trabajo tan arduo y extenso. 
Además, el libro es un estudio sobre una dimensión trabajada tradicionalmente desde la óptica de los propios países donde ocurre el fenómeno, y en este caso el lente con que se mira el problema proviene de un país desarrollado, lo cual sin duda tiene implicaciones que son interesantes de analizar.

Existe mucha información importante en el libro, pero destacan tres elementos que considero fundamentales, el primero es el abordaje del fenómeno de la pobreza y sus repercusiones medido a partir de técnicas econométricas, sustentadas en datos empíricos. El segundo, la voz de los pobres refiriéndose a sus emociones, aspiraciones y desencantos relacionados con el desarrollo del turismo como actividad productiva en cada una de sus regiones y por último, la propuesta de los autores para la reducción de la pobreza, la cual es sin duda es bastante particular y digna de analizar más en detalle.

La invitación queda hecha, la lectura del libro resultará muy interesante y sin duda generará muchos cuestionamientos, interrogantes y nuevas líneas de investigación, lo cual es el mayor logro de todo trabajo de investigación; despertar la curiosidad y convertirse en nueva fuente de conocimiento. 